\title{
Efficacy and Safety Profile of Risperidone Long-acting Injection in Adolescents in a Real-life Setting
}

\author{
Ulku Akyol Ardic ${ }^{1}$, Mustafa Küçükköse ${ }^{2}$, Sevim Berrin Inci ${ }^{3}$, Eyüp Sabri Ercan ${ }^{4}$ \\ ${ }^{1}$ Department of Child and Adolescent Psychiatry, Denizli State Hospital, Denizli, ${ }^{2}$ Department of Child and Adolescent Psychiatry, Aydin State \\ Hospital, Aydin, ${ }^{3}$ Institute on Drug Abuse, Toxicology and Pharmaceutical Science, Ege University, Izmir, ${ }^{4}$ Department of Child and Adolescent \\ Psychiatry, Ege University Faculty of Medicine, Izmir, Turkey
}

\begin{abstract}
Objective: Risperidone long-acting injection (RLAl) was shown to be an alternative option in adult patients, but there is not available data in child and adolescents about this medication. The aim of this study is to evaluate the safety and efficiency profile of RLAI in a group of adolescents.

Methods: Eleven cases with conduct disorder and severe aggressive behaviors were initiated treatment with risperidone oral form. All cases were then shifted to RLAI $25 \mathrm{mg}$ injection in each 15 days due to poor compliance to oral risperidone treatment. Efficiency of treatment included indicators of clinical severity and improvement, which were evaluated by Clinical Global Impression-Severity (CGI-S) and Improvement (CGI-I). Safety evaluation was performed by using Extrapyramidal Symptoms Rating Scale, and by monitoring body weight. Follow-up visits were done at the treatment initiation, and 8th, 16th, and 24th weeks of first injection.

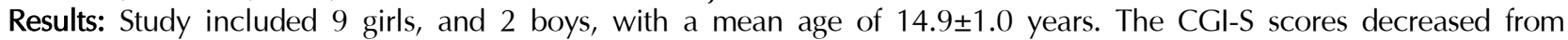
$6.6 \pm 0.5$ at the beginning to $2.2 \pm 1.1$ at the last visit $(p<0.001)$, which is a very significant decrease through better clinical level. The CGI-I scores were also improved significantly from $2.4 \pm 0.5$ to $1.9 \pm 0.5$ at 24 th week $(p=0.001)$. Safety parameters were also showed favorable results, which there was no significant weight gain $(p=0.076)$, and well-tolerated extrapyramidal adverse effects.

Conclusion: Our results showed that RLAI is an efficient and safe medication option in the treatment of psychiatric disorders and severe behavioral problems in adolescents with low-compliance to oral treatment in our cases.
\end{abstract}

KEY WORDS: Risperidone; Risperidone long-acting injection; Adolescent; Conduct disorder.

\section{INTRODUCTION}

Aggressive behaviors are disorders that may develop in very early stages of life, even in infancy, ${ }^{1)}$ and may persist through childhood and adolescence. ${ }^{2)}$ The course of aggressive behaviors has individual differences, ${ }^{3)}$ which may be related with the underlying psychiatric disorder. Advanced levels of aggression are among the leading disorders that require intense psychopharmacological intervention, because they may progress to violent behaviors in adolescence. ${ }^{4)}$ Diagnostic and Statistical Manual of

Received: November 14, 2016/Revised: March 23, 2017 Accepted: May 22, 2017

Address for correspondence: Ulku Akyol Ardic, MD Department of Child and Adolescent Psychiatry, Denizli State Hospital, Merkez Efendi Street, Denizli 20100, Turkey

Tel: +90-505-398-85-65, +90-232-390-14-07, Fax: +90-232-

390-13-50

E-mail: ulkuakyol@yahoo.com
Mental Disorders, 5th edition (DSM-5) cites childhood aggressive behavior as a criterion for disruptive disorders including oppositional defiant disorder and conduct disorder (CD). ${ }^{5)}$ Additionally, some other neurodevelopmental diseases such as attention deficit hyperactivity disorder (ADHD), autism spectrum disorder, dysregulated behaviors, substance use disorders, antisocial personality disorders and schizophrenia also includes clinical components of aggressive behaviors during their course. ${ }^{6-11)}$

Current evidence suggests that most frequently preferred medication in treatment of this disorder is atypical antipsychotics. Risperidone is being used with a growing frequency in the child and adolescent psychiatry practice in USA and Europe. A recent report in 2014 about the Pediatric European Risperidone Studies declared that it might provide efficient treatment results in this patient population. ${ }^{12)}$ Nevertheless, medicating adolescents is

(ㄷ) This is an Open-Access article distributed under the terms of the Creative Commons Attribution Non-Commercial License (http://creativecommons.org/licenses/by-nc/4.0) which permits unrestricted non-commercial use, distribution, and reproduction in any medium, provided the original work is properly cited. 
frequently challenged by pitfalls such as treatment refusal or lack of compliance to proper usage of the drugs. Using liquid forms of atypical antipsychotics, like zuclopentiol, may partly overcome medication refusal. ${ }^{13)}$ But, this approach also has some challenges, including the difficulty of dose adjustment, and refusal of foods and drinks by some adolescents when they realize that they are being medicated without notice.

Risperidone long-acting injection (RLAI) may be another alternative in cases of these challenges. Previous studies suggested that depot formulations of atypical antipsychotics both exhibit the advantages of pill formulations, and also the stabilization of serum drug levels. ${ }^{14)}$ But, currently there is no available data about the use of RLAl in child and adolescent population with severe aggression.

Under the light of the above scientific evidences, the aim of current study was determined as evaluating the outcomes of RLAl treatment in a group of adolescents, who exhibit severe aggressive symptoms and experience disadvantages of academic and social failures due to incompliance to their treatment.

\section{METHODS}

In this retrospective study, data of 11 adolescents were included, who all have been treated between 2014 and 2015 in the child and adolescent psychiatry outpatient clinic of the State Hospital of Denizli, in the western Turkey. After obtaining local approval of the protocol, the patient data were traced forward from the clinical follow-ups, since initiation of RLAI until the preparation of the manuscript. Demographic, clinical and treatment characteristics of the patients were reported regarding the efficiency and safety of RLAI, including clinical outcomes and side effects, respectively.

\section{Cases}

Eleven adolescents, whom all were referred to the department with aggressive behaviors, were included in the study. Patients initially diagnosed with $\mathrm{CD}$ by a senior child and adolescent psychiatrist, and prescribed atypical antipsychotics. During the follow-ups, cases reported clinical benefit from oral risperidone treatment, but the efficiency was partial due to incompetence with the drug regimen. Partial efficiency was defined as improvement of symptoms at the periods with appropriate use of drugs, but deteriorations in inconsistent use of medications. Consequently, parents were informed about the potential clinical benefits of RLAI, and after obtaining the consents all patients shifted to a regimen of $25 \mathrm{mg}$ of intramuscular injection of RLAI per 15 days of cycles.

\section{Follow-up and Clinical Assessment}

At the beginning of treatment, patients were evaluated in clinical visits at the days of RLAI injection. Once the clinical benefits were documented through clinical assessment scales, the frequencies of follow-ups were elongated to 60 days. The improvements in aggressive behaviors were rated by using Clinical Global ImpressionSeverity of Illness (CGI-S) and Improvement (CGI-I) scales. Guy ${ }^{15)}$ first described these scales for the clinical follow-up of psychiatric disorders at any age group. The CGI-S is rated on a 7-point scale, with the severity of illness scale using a range of responses from 1 (normal) through to 7 (amongst the most severely ill patients). CGI-I scores range from 1 (very much improved) through to 7 (very much worse). ${ }^{15)}$ In our patient group, CGI-S was administered at 8th, 16th, and 24th weeks, and CGI-I was administered at each follow-up interview but the first visit.

Since no adverse events other than weight gain and extrapyramidal symptoms were reported, safety evaluation of RLAI included weight gain and extrapyramidal adverse event examination. Extrapyramidal system evaluation was performed by using Extrapyramidal Symptom Rating Scale, which was designed by Chouinard and Margolese. ${ }^{16)}$

\section{Statistical Analyses}

Numerical data were presented as mean and standard deviation, and categorical variables were shown as frequencies and percent in descriptive analyses. Changes in CGI scores and body weight during treatment period were compared with Friedman non-parametric analysis of variances. Post-hoc pairwise comparisons were performed by Wilcoxon signed-ranks test with Bonferroni correction. All statistical analyses were two-tailed with a type-I error level of 5\%. SPSS Statistics ver. 21.0 (IBM Co., Armonk, NY, USA) was used for the analyses in the study.

\section{RESULTS}

Nine of the patients were female (mean age, $15.1 \pm 0.9$ 
Table 1. Demographic and clinical characteristics of patient group

\begin{tabular}{lcc}
\hline \multicolumn{1}{c}{ Characteristic } & Data & $p$ value \\
\hline Age (yr) & & 0.727 \\
Girls & $15.1 \pm 0.9$ & \\
Boys & $14.5 \pm 2.1$ & \\
CGl-Severity & & $<0.001$ \\
Treatment onset* & $6.55 \pm 0.52$ & \\
8th week & $3.09 \pm 0.94$ & \\
16th week & $2.45 \pm 0.52$ & \\
24th week & $2.18 \pm 1.08$ & \\
CGI-Improvement & & 0.001 \\
8th week & $2.55 \pm 0.69$ & \\
16th week & $2.36 \pm 0.51$ & \\
24th week* & $1.91 \pm 0.54$ & \\
\hline
\end{tabular}

Values are presented as mean \pm standard deviation.

CGI, Clinical Global Impression.

*The significantly distinct group in multiple comparisons.

years) and 2 were male (mean age, $14.5 \pm 2.1$ years), and mean ages of both sexes were similar $(p=0.727)$. The efficacy analyses were performed by analyzing CGI scale scores during follow-up visits. At the initiation of RLAI treatment the severity of the patients' disorders shown by CGI-S was 6.6 60.5 (range, 6-7), which corresponds a severe disease. During the follow-up, the CGI-S scores decreased gradually to $2.2 \pm 1.1$ at the last visit, and this downward trend was statistically significant $(p<0.001)$. While the severity of the disease decreases during the treatment, improvements in clinical symptoms were also significant. The CGI-I measurements at 8th week showed that the mean score was $2.4 \pm 0.5$, and this score decreased to $1.9 \pm 0.5$ at 24 th week. The improvement of these scores during follow-ups was also statistically significant ( $p=0.001)$. The age, and CGI-S and CGI-I scores were shown in Table 1.

The safety analyses of RLAI treatment included evaluation of weight gain and occurrence of extrapyramidal symptoms. The mean body weight at the beginning of treatment was $55.5 \pm 5.7 \mathrm{~kg}$, and during follow-ups, it shifted to $57.0 \pm 5.7 \mathrm{~kg}$. The change through the treatment was not statistically significant $(p=0.076)$. While there is no significant change in body weight, the extrapyramidal symptoms were also improved during treatment course. At the 8th week visits, only 2 patients tolerated the drug without any extrapyramidal adverse events (18.2\%), but 6 patients had no extrapyramidal symptoms in 24th week follow-up visits (54.5\%). The complete list of the adverse events was shown in Table 2.
Table 2. Indicators of safety of risperidone long-acting injection treatment

\begin{tabular}{ll}
\hline \multicolumn{1}{c}{ Variable } & Data \\
\hline Body weight $(\mathrm{kg})^{*}$ & \\
Treatment onset & $55.5 \pm 5.7$ \\
8th week & $56.8 \pm 5.5$ \\
16th week & $56.5 \pm 5.2$ \\
24th week & $57.0 \pm 5.7$ \\
Extrapyramidal symptoms & \\
8th week & \\
None & $2(18.2)$ \\
Mild sedation & $6(54.5)$ \\
Moderate sedation & $2(18.2)$ \\
Moderate sedation+mild stiff posture & $1(9.1)$ \\
16th week & \\
None & $5(45.5)$ \\
Mild sedation & $4(36.4)$ \\
Mild sedation+mild stiff posture & $1(9.1)$ \\
Mild sedation+mild speech difficulty & $1(9.1)$ \\
24th week & \\
None & $6(54.5)$ \\
Mild sedation & $3(27.3)$ \\
Mild sedation+mild speech difficulty & $1(9.1)$ \\
Mild sedation+mild stiff posture & $1(9.1)$ \\
\hline
\end{tabular}

Values are presented as mean \pm standard deviation or number (\%). ${ }^{*} p=0.076$.

\section{DISCUSSION}

To the best of our knowledge, this is the first study with a patient group that reports the efficiency and safety of RLAI in adolescents who diagnosed with CD and exhibited severe aggressive behaviors. The only data about RLAI in adolescents was from a previous case report by Tutkunkardaş and Abali, ${ }^{17)}$ which reported that RLAI was efficient in the treatment of an adolescent with ADHD and CD. In our study, the patients were primarily treated with risperidone oral pills, but shifted to an intramuscular long-acting atypical antipsychotic regimen due to failure of treatment compliance. The efficacy analyses showed that clinical symptoms were successfully improved during 24 weeks of RLAl treatment, which was shown by CGI scale assessments. Meanwhile this efficiency, RLAI treatment was also shown to be a safe medication, which was evaluated by weight gain and extrapyramidal symptoms.

There are many recent reports about the efficiency of atypical antipsychotic agents in the management of psychiatric disorders. Peruzzolo et al. ${ }^{18)}$ reported the efficiency in children and adolescents with bipolar disorder. Ercan et al. ${ }^{13,19)}$ showed in their two studies that risper- 
idone was effective in the treatment of $C D$ in children. And, another study by Research Units on Pediatric Psychopharmacology Autism Network reported that risperidone was effective and well tolerated for the treatment of tantrums, aggression, or self-injurious behavior in children with autistic disorder. ${ }^{20)}$ But, efficiency of risperidone is hampered by the lack of long-term treatment compliance to the oral form of this drug. ${ }^{21)}$

One possible way to overcome this barrier is using long-acting forms of atypical antipsychotics in the treatment of non-compliant cases. In a prospective study of Vieta et al., ${ }^{22)}$ RLAl was shown to significantly decrease the compliance failures, also enhanced compliance to the other medications. Meanwhile increasing the compliance, Macfadden et al. ${ }^{23)}$ and Quiroz et al. ${ }^{24)}$ showed that RLAl also decreased the relapse in bipolar disorder when used with other treatments or as monotherapy. But, a literature search about the use of RLAI in the treatment of psychiatric disorders in child and adolescents with severe aggressive behaviors returned no results. Only study about the use of RLAl in child and adolescents was conducted by Boarati et al., ${ }^{25)}$ which included patients with pediatric bipolar disease. This study reported that RLAI might be an alternative treatment in children who have not responded to medication or who had problems with treatment adherence. According to our results, RLAI is an efficient and safe treatment also for CD and behavioral problems, like severe aggressive behaviors in our cases. Decreases in CGI-S scores, and enhancement in CGI-I scores showed the efficacy of this medication.

As well as the efficiency of RLAl in adolescents, it was also shown to be a safe treatment modality. We observed neither any significant weight gain, nor any severe or intolerable extrapyramidal adverse events in our cases. The most common adverse events of atypical antipsychotic agents are known to be weight gain, loss of glycemic control, dyslipidemia and hyperprolactinemia. ${ }^{26)}$ Previous studies by Macfadden et al. ${ }^{23)}$ and Boarati et al. ${ }^{25)}$ reported that their cases had about $2 \mathrm{~kg}$ and $5.7 \mathrm{~kg}$ of increases in body weight, respectively. Despite these studies, our cases had no significant weight gain during the 24 week of treatment.

Another safety related clinical indicator was the occurrence of extrapyramidal symptoms in our study. According to our findings nearly half of the patients had mild sedation, and about $20 \%$ of patients had moderate sedation at the 8th week of treatment initiation. One of the most comprehensive studies about the use of risperidone in children, which conducted by Research Units on Pediatric Psychopharmacology Autism Network, reported a mild sedation rate of $59 \%$ in their series, ${ }^{20)}$ which is comparable to our results. Although the medication in that study was oral form of risperidone, but RLAI in our study also exhibited a well-tolerated safety profile, even the tolerability was increased during treatment and more than half of the patients had no extrapyramidal adverse events at the 24th week.

This is the first study that showed the efficiency of RLAI in the treatment of conduct disorder and severe aggressive behavioral problems in adolescents. Previous findings about the oral form of risperidone were favoring this treatment in psychiatric disorders of youngsters, but there was no available data about RLAI in this age group. Our results suggest that RLAI form is a safe, well-tolerated and efficient treatment option in adolescents.

\section{- Acknowledgments}

Eyüp Sabri Ercan is in charge of the advisory board of Lilly and Janssen-Cilag, While undertaking this study, no support was received. The authors declare no competing financial interests.

Dr. Ulku Alyol Ardic designed the study and wrote the first draft. Dr. Eyüp Sabri Ercan conducted statistical analysis. Dr. Mustafa Küçükköse and Sevim Berrin Inci assisted with the literature searches. All authors contributed to and have approved the final manuscript.

\section{REFERENCES}

1. Lewis M, Alessandri SM, Sullivan MW. Violation of expectancy, loss of control, and anger expressions in young infants. Dev Psychol 1990;26:745-751.

2. Alink LR, Mesman J, van Zeijl J, Stolk MN, Juffer F, Koot HM, et al. The early childhood aggression curve: development of physical aggression in 10- to 50-month-old children. Child Dev 2006;77:954-966.

3. Tremblay RE, Nagin DS, Séguin JR, Zoccolillo M, Zelazo PD, Boivin M, et al. Physical aggression during early childhood: trajectories and predictors. Can Child AdolesC Psychiatr Rev 2005; 14:3-9.

4. Raine A. Biosocial studies of antisocial and violent behavior in children and adults: a review. I Abnorm Child Psychol 2002;30:311-326.

5. American Psychiatric Association. Diagnostic and statistical 
manual of mental disorders: DSM-5. 5th ed. Arlington, VA:American Psychiatric Publishing;2013.

6. Hamshere ML, Langley K, Martin J, Agha SS, Stergiakouli E, Anney RJ, et al. High loading of polygenic risk for $A D H D$ in children with comorbid aggression. Am J Psychiatry 2013; 170:909-916.

7. Mandy W, Roughan L, Skuse D. Three dimensions of oppositionality in autism spectrum disorder. J Abnorm Child Psychol 2014;42:291-300.

8. Pope AW, Bierman KL. Predicting adolescent peer problems and antisocial activities: the relative roles of aggression and dysregulation. Dev Psychol 1999;35:335-346.

9. White HR, Fite P, Pardini D, Mun EY, Loeber R. Moderators of the dynamic link between alcohol use and aggressive behavior among adolescent males. J Abnorm Child Psychol 2013; 41:211-222.

10. Schaeffer $\mathrm{CM}$, Petras $\mathrm{H}$, lalongo $\mathrm{N}$, Poduska J, Kellam S. Modeling growth in boys' aggressive behavior across elementary school: links to later criminal involvement, conduct disorder, and antisocial personality disorder. Dev Psychol 2003; 39:1020-1035.

11. Volavka J, Bilder R, Nolan K. Catecholamines and aggression: the role of COMT and MAO polymorphisms. Ann N Y Acad Sci 2004; 1036:393-398.

12. Glennon J, Purper-Ouakil D, Bakker M, Zuddas A, Hoekstra P, Schulze U, et al. Paediatric European Risperidone Studies (PERS): context, rationale, objectives, strategy, and challenges. Eur Child Adolesc Psychiatry 2014;23:1149-1160.

13. Ercan ES, Basay BK, Basay O, Durak S, Ozbaran B. Risperidone in the treatment of conduct disorder in preschool children without intellectual disability. Child Adolesc Psychiatry Ment Health 2011;5:10.

14. Kane JM, Eerdekens M, Lindenmayer JP, Keith SJ, Lesem M, Karcher K. Long-acting injectable risperidone: efficacy and safety of the first long-acting atypical antipsychotic. Am J Psychiatry 2003;160:1125-1132.

15. Guy W. ECDEU assessment manual for psychopharmacology. Rockville, MD:US Department of Health, Education, and Welfare; Public Health Service, Alcohol, Drug Abuse, and Mental Health Administration; National Institute of Mental Health, Psychopharmacology Research Branch, Division of Extramural Research Programs; 1976.
16. Chouinard G, Margolese HC. Manual for the Extrapyramidal Symptom Rating Scale (ESRS). Schizophr Res 2005;76:247265.

17. Tutkunkardaş MD, Abali O. Long acting risperidone in an adolescent with conduct disorder: A case report. Psychopharmacol Bull 2011;44:69-72.

18. Peruzzolo TL, Tramontina S, Rohde LA, Zeni CP. Pharmacotherapy of bipolar disorder in children and adolescents: an update. Rev Bras Psiquiatr 2013;35:393-405.

19. Ercan ES, Kutlu A, Cikoğlu S, Veznedaroğlu B, Erermiş $S$, Varan A. Risperidone in children and adolescents with conduct disorder: a single-center, open-label study. Curr Ther Res Clin Exp 2003; 64:55-64.

20. McCracken JT, McGough J, Shah B, Cronin P, Hong D, Aman $\mathrm{MG}$, et al. Risperidone in children with autism and serious behavioral problems. N Engl J Med 2002; 347:314-321.

21. Macfadden W, Adler CM, Turkoz I, Haskins JT, Turner N, Alphs L. Adjunctive long-acting risperidone in patients with bipolar disorder who relapse frequently and have active mood symptoms. BMC Psychiatry 2011;11:171.

22. Vieta E, Nieto E, Autet A, Rosa AR, Goikolea JM, Cruz N, et al. A long-term prospective study on the outcome of bipolar patients treated with long-acting injectable risperidone. World J Biol Psychiatry 2008;9:219-224.

23. Macfadden W, Alphs L, Haskins JT, Turner N, Turkoz I, Bossie $\mathrm{C}$, et al. A randomized, double-blind, placebo-controlled study of maintenance treatment with adjunctive risperidone long-acting therapy in patients with bipolar I disorder who relapse frequently. Bipolar Disord 2009;11:827-839.

24. Quiroz JA, Yatham LN, Palumbo JM, Karcher K, Kushner S, Kusumakar V. Risperidone long-acting injectable monotherapy in the maintenance treatment of bipolar I disorder. Biol Psychiatry 2010;68:156-162.

25. Boarati MA, Wang YP, Ferreira-Maia AP, Cavalcanti AR, Fu-I L. Six-month open-label follow-up of risperidone long-acting injection use in pediatric bipolar disorder. Prim Care Companion CNS Disord 2013;15:PCC.12m01368.

26. Jerrell JM, McIntyre RS, Tripathi A. Childhood treatment with psychotropic medication and development of comorbid medical conditions in adolescent-onset bipolar disorder. Hum Psychopharmacol 2011;26:451-459. 